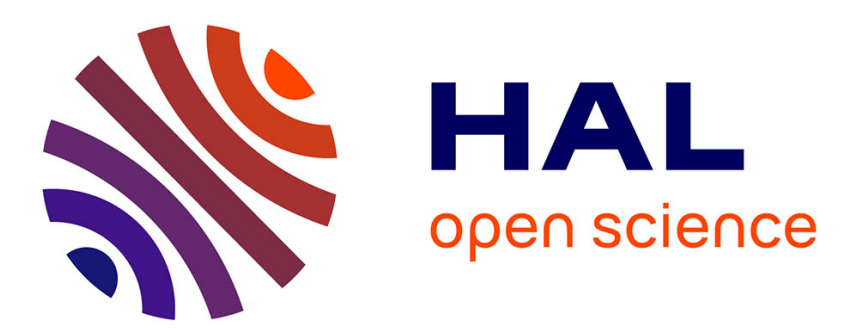

\title{
Driver-aid system using path-planning for lateral vehicle control
}

Jean-Philippe Lauffenburger, Michel Basset, Frank Coffin, Gérard Gissinger

\section{To cite this version:}

Jean-Philippe Lauffenburger, Michel Basset, Frank Coffin, Gérard Gissinger. Driver-aid system using path-planning for lateral vehicle control. Control Engineering Practice, 2003, 11, pp.217-231. 10.1016/S0967-0661(02)00047-3 . hal-00933588

\section{HAL Id: hal-00933588 \\ https://hal.science/hal-00933588}

Submitted on 24 Jan 2014

HAL is a multi-disciplinary open access archive for the deposit and dissemination of scientific research documents, whether they are published or not. The documents may come from teaching and research institutions in France or abroad, or from public or private research centers.
L'archive ouverte pluridisciplinaire HAL, est destinée au dépôt et à la diffusion de documents scientifiques de niveau recherche, publiés ou non, émanant des établissements d'enseignement et de recherche français ou étrangers, des laboratoires publics ou privés. 


\title{
Driver-aid system using path-planning for lateral vehicle control ts
}

\author{
J.Ph. Lauffenburger ${ }^{\mathrm{a}, *}$, M. Basset ${ }^{\mathrm{a}}$, F. Coffin ${ }^{\mathrm{b}}$, G.L. Gissinger ${ }^{\mathrm{a}}$ \\ ${ }^{\mathrm{a}}$ MIPS Laboratory, Department of Automotive Control (MIAM-ESSAIM), University of Haute-Alsace, 12 rue des frères Lumière 68093 , \\ Mulhouse, France \\ ${ }^{\mathrm{b}}$ RENAULT Research Department, CTA-Parc de Gaillon-G13, F-27940 Aubevoye, France
}

Received 19 October 2001; accepted 17 January 2002

\begin{abstract}
The Navigation Aided Intelligent Cruise Control (N.A.I.C.C.) system acts in the field of the lateral and longitudinal control of a car. This paper only describes the lateral part of this new driver-aid system. Once the vehicle is located on the road, it determines the distance to the next bend and predicts a reference path to negotiate this bend considering the road profile, the vehicle characteristics and the driver's profile. The use of polar curves for the description of a turn eliminates steering function discontinuities by providing continuous curvature along the path. The computation of the polynomial is based on a fuzzy-logic method which determines the characteristics of the end-points of the polynomial curve. In order to compute realistic paths, real experiments were carried out with the instrumented laboratory test car. (C) 2003 Elsevier Science Ltd. All rights reserved.
\end{abstract}

Keywords: Trajectory modelling; Polar polynomials; Intelligent driver-aid system; Real-time; Fuzzy logic

\section{Introduction}

Ever increasing traffic forces the driver to process a growing amount of information and, at the same time, to take more, and quicker, decisions. Thus, in critical situations, the amount of information may exceed the driver's effective processing capability. Thanks to the improvements in computer technologies and in mechatronics, the driver can now be assisted in the detection and/or correction of a critical situation with the help of on-board systems integrated in the driver-vehicle loop (Onken, 1993; An \& Harris, 1996). This technique which forms part of active safety has become one of the principal research activities in the automotive industry. The current strategy adopted by car manufacturers as well as psychologists in the development of active

\footnotetext{
An earlier shorter version of this paper entitled "a vehicle pathplanning algorithm using polar polynomials optimised through fuzzy logic" was presented at the First IFAC Conference on Mechatronic Systems in Darmstadt, Germany, September 2000.

*Corresponding author. Tel.: + 33-3-89-33-69-41; fax: + 33-3-89-3369-49.

E-mail addresses: j.lauffenburger@uha.fr (J.Ph. Lauffenburger), m.basset@uha.fr (M. Basset), f.coffin@renault.com (F. Coffin), g.gissinger@uha.fr (G.L. Gissinger).
}

systems consists in replacing the driver for the simplest tasks and in assisting him as much as possible for the more complicated ones. The principle is based on the minimisation of the direct interactions between the driver and the vehicle and the maximisation of those between the driver-aid system (also called copilot) and the vehicle. Safety systems and more particularly copilots which are currently under development and will be available in the next decades (Shladover, 1998) cover a large application field (Roser \& Moissidis, 1998): curve warning systems, adaptive speed regulation systems, stability control systems. Then, in this context, a human-machine or driver-copilot cooperation structure has to be clearly defined in order to integrate the copilot into the driver-vehicle loop. Two generic structures can be considered: the vertical (hierarchical) and the horizontal (heterarchical) structures. In the hierarchical scheme, the copilot only gives advice to guide the driver in his decisions whereas in the heterarchical model, the copilot and the driver can both take the decision since they are on the same hierarchical level in the decision structure (Millot, 1998; Yuhara et al., 1998).

In the field of intelligent assistance systems, the Navigation Aided Intelligent Cruise Control (N.A.I.C.C.) project has been initiated to improve the 
driver's safety and comfort on any type of roads, mainly on open roads. In this framework, the aim of the N.A.I.C.C. project is:

- in the first step, to detect and warn the driver in the case of inappropriate driving conditions in terms of speed and/or trajectory. This open-loop mode is known as "Driver Warning Mode",

- in the second step, when used in its closed-loop mode ("Vehicle Control Mode"), to detect and correct these inappropriate driving conditions considering the profile of the road (bend, ...) and the characteristics given by the driver.

This paper describes the results obtained using the N.A.I.C.C. lateral part (trajectory estimation for control) as a warning system. In this configuration, N.A.I.C.C. computes a reference path for a given driving situation and warns the driver if the real (measured) vehicle trajectory deviates too much from this reference.

The principle used for trajectory estimation is derived from the path-planning technique for autonomous guided vehicles in robotics. Traditionally, the generated paths for routing these kinds of robots are composed of a succession of line segments and circular-arc segments. An attempt to follow such paths results in discontinuities in the steering functions at each junction between the straight lines (of zero curvature) and the circular arcs (of constant curvature equal to the inverse of the arc radius). The use of continuous curvature curves allowing zero curvature at their end-points can solve this problem. Applied to the N.A.I.C.C. system, the goal of this technique is to determine a reference trajectory that best fits to the driver's style (novice, experienced...). Assuming that the N.A.I.C.C. integrated navigation system precisely locates the vehicle on a digital map database, this intelligent driver-aid system predicts a smoothly curved reference path, taking account of the information provided by the database. Afterwards, it warns the driver if he is deviating from the real-time computed trajectory. The computed path is based on a polynomial representation of its radius of curvature with respect to the turn angle and is called polar polynomial. The parameters of the polar curve describing the trajectory are obtained via a fuzzy inference system (FIS) considering the road characteristics contained in the database.

After a brief presentation of the research tools (test car and test track) and of the N.A.I.C.C. structure, this paper addresses, in the first part, the properties of the polar polynomial, the reasons of this choice and, in the second part, the fuzzy determination of the parameters. It will then present the results, on the one hand, of path modelling in a bend and, on the other hand, of the pathplanning module used for path generation in a succes- sion of straight lines and curves. The conclusion will consider the perspectives of this project.

\section{Measurements}

All the experiments were carried out with the instrumented laboratory test car (a Renault Megane $2.0 \mathrm{~L} 16 \mathrm{~V}$ ) on a test track to ensure maximum safety.

\subsection{The test car}

\subsubsection{Currently available sensors}

The different sensors and measurement parameters needed are shown in Fig. 1. The longitudinal and lateral velocities $\left(V_{L}, V_{T}\right)$ are measured during the validation period of the N.A.I.C.C. system by an optical cross-correlation sensor located at the rear centre of the vehicle. The piezoelectric vibrating gyroscope, the microprocessor-controlled fluxgate compass and the accelerometers are located near the centre of gravity of the test car. They measure, respectively, the yaw rate $(\dot{\psi})$, the yaw angle $(\psi)$, the longitudinal and transverse accelerations $\left(\gamma_{L}, \gamma_{T}\right)$. During the tests, the throttle valve angle $\left(\theta_{P a p}\right)$ and the steering-wheel angle $\left(\alpha_{V}\right)$ are also measured.

\subsubsection{The DGPS system}

The global positioning system (GPS) is a satellitebased radio-navigation system which determines the absolute position with an accuracy ranging from $100 \mathrm{~m}$ (Zhao, 1997) to some centimetres (Gounon \& Barboux, 1997), depending on the technologies used and application fields. The use of two GPS receivers allows more accurate position determination. One receiver is used as a static reference with known coordinates. It evaluates a correction (difference between its known position and

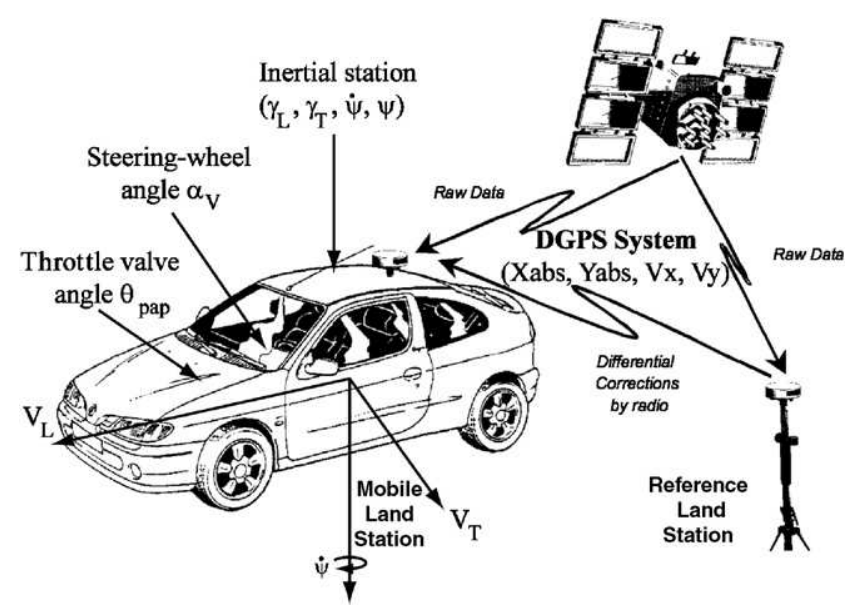

Fig. 1. Sensors and measurement parameters in the instrumented laboratory car. 
the one it is currently measuring using the satellites information) and sends the information to the mobile station located on the car. This technique is known as differential GPS (DGPS) and reduces the position error to $10^{-2} \mathrm{~m}$ for the DGPS used in this research work. This precision is particularly interesting and is required when studying different driver profiles in terms of their respective trajectories. On the other hand, a precision of some metres can be enough in the case of the integration of a GPS device in a navigation system.

\subsubsection{Acquisition hardware}

The real-time data acquisition hardware is based on a DSP board and I/O boards linked via Ethernet to a PC in which the data are stored. A sampling frequency of $50 \mathrm{~Hz}$ has been chosen for all the sensors, except for the DGPS and the microprocessor-controlled fluxgate compass working at a $10 \mathrm{~Hz}$ frequency.

\subsection{The test track}

The bird's-eye view plotted in Fig. 2 describes the track used to develop and validate the N.A.I.C.C. system. This track is composed of bends whose radii of curvature cover a large field of possibilities (from 30 to $150 \mathrm{~m}$ ). During the tests, only the so-called "slow part" of the track has been used.

\section{The N.A.I.C.C. system}

The N.A.I.C.C. project includes two research activities: the longitudinal and the lateral control of a car. Two operating modes are proposed: the "Driver Alarm Mode" presented here and the "Control Mode". In the first configuration, this driver-selectable system warns the driver of a critical situation detection in terms of inappropriate speed and/or trajectory, whereas in the second configuration, the system corrects the speed (longitudinal part) and/or the trajectory (lateral part), considering a computed reference. Fig. 3 describes the two control schemes of the N.A.I.C.C.. This driver-aid system is composed of a location module which identifies the vehicle on the database. Using the determined location and the road characteristics stored in the database, N.A.I.C.C. determines a reference speed and/or path for a given driving situation. Fig. 3 shows the importance of the navigation part (the location and the environment detection modules) common to the longitudinal and lateral control loops. A precise vehicle location as well as precise information on the current environment (highway, town...) in which the vehicle is moving is required to perform the lateral or even the longitudinal control tasks. Data fusion techniques based on the information provided by the DGPS and the "traditional" sensors mounted on the vehicle have been developed to determine the location of the vehicle accurately.

Even if the lateral and the longitudinal control schemes have been represented and specified separately in this figure, it is obvious that they are closely linked. Considering a given trajectory, the achievable longitudinal velocity depends on the vehicle's position along the path.

\subsection{The N.A.I.C.C. longitudinal controller}

This controller predicts the reference longitudinal speed for a given driving situation, once the vehicle has been located on the digital map database (Lauffenburger, Baujon, Basset, \& Gissinger, 2000). In this case, it can be considered as a generalisation of the speed regulation system (cruise control) applied to open roads and not only on highways: the driver activates the system which automatically adapts the vehicle's speed to the road profile (bend negotiation) or the detected driving situation (obstacle detection...).

\subsection{The N.A.I.C.C. lateral controller}

The aim of the lateral part is to compute a reference trajectory that the driver should take to perform a given driving manoeuvre (curve negotiation), taking account of the vehicle location, the road characteristics and the constraints given by the driver. Afterwards, the first step consists in the determination of the deviation between the real trajectory and the computed one. Then, in the

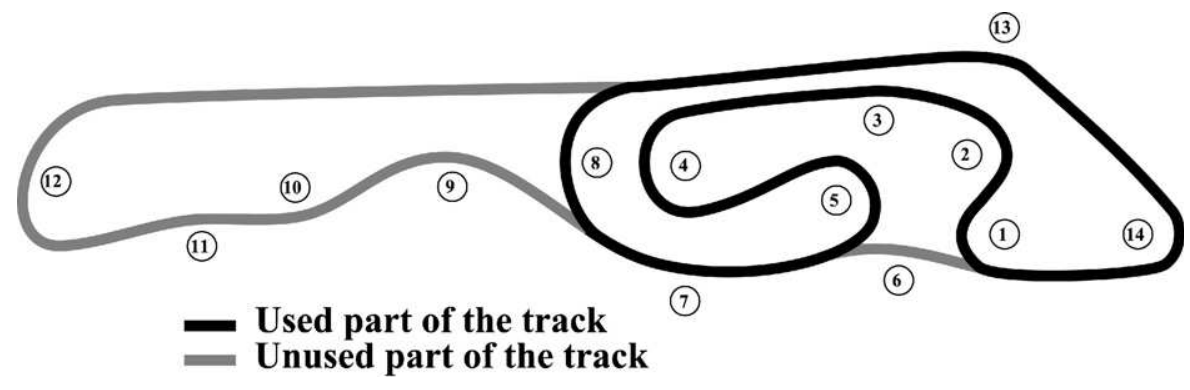

Fig. 2. The test track. 


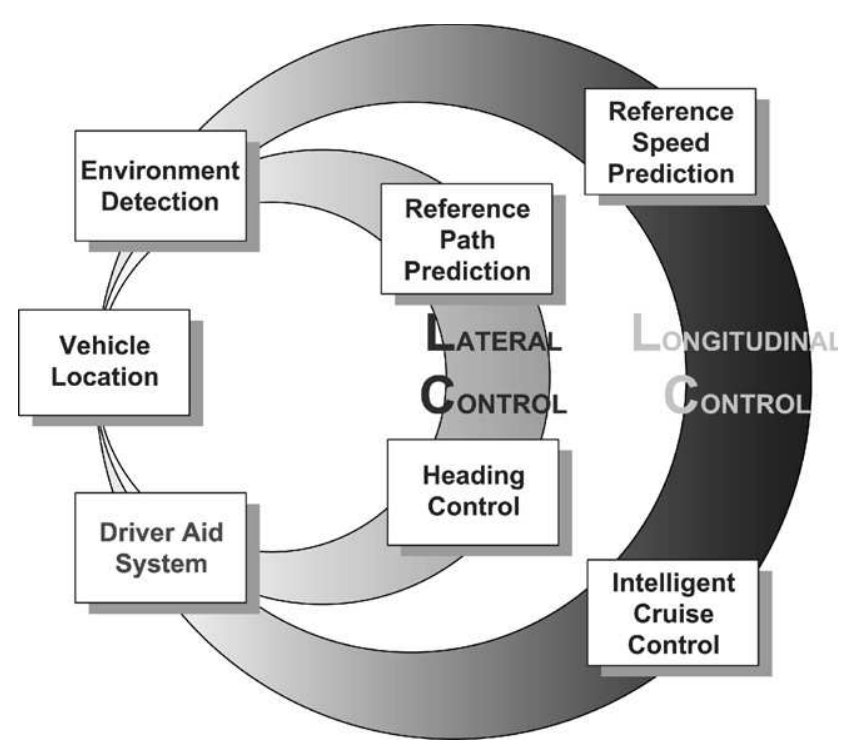

Fig. 3. Structure of the N.A.I.C.C. system.

"Warning Mode", the system informs the driver that he is deviating from the reference path. The second step consists in the correction ("Vehicle Control Mode") of the vehicle's trajectory if the deviation is too important.

\section{Polar polynomials}

Knowing a precise location of the vehicle on the digital database, on the one hand, and the driver's profile (novice, experienced...), on the other hand, the system must be able to determine a reference trajectory which corresponds to the one the driver would take in normal conditions. When an "inexperienced driver", as defined by Rothengatter, Alm, Kuiken, Michon, and Verwey (1993), negotiates a bend, he smoothly increases the steering angle during the first half of the turn and smoothly decreases it during the second half (see Section 5.1). This driving technique is guided by the search for maximum comfort and safety (minimisation of the lateral speed and lateral acceleration variations). For an "experienced driver", the trajectory in the same turn is completely different (see Section 5.2) because his objectives are different (time minimisation...).

In the first stage, the goal of this study is to model and identify the trajectory characteristics of different kinds of drivers. In the second stage, the aim is to use these characteristics to define a realistic path-planning algorithm which reproduces trajectories corresponding to the identified driver profiles.

The mathematical model used here is based on a representation of the trajectory in a polar coordinate frame (radius, polar angle), expressing the radius of the path $r$ with a polynomial relation with respect to the polar angle $\phi$. The polar polynomial curves were introduced by Nelson $(1989 \mathrm{a}, \mathrm{b})$ in the case of path- planning for autonomous robots. Generally, the paths were sequences of straight lines and arcs of circles. Unfortunately, these paths produce discontinuities in curvature, and thus in the centrifugal acceleration, at the line-arc transitions. To avoid these discontinuities, Nelson developed a fifth-order polynomial with continuous curvature that approximates as far as possible the arc of circle used initially. The coefficients are calculated, imposing conditions on the position (the radius), the slope (the derivative of the radius with respect to the polar angle) and on the curvature at the extremes of the curve. These six conditions allow perfect (error-free) matching with the line-arcs at each junction. Considering the N.A.I.C.C. project, an interesting characteristic of these curves is that the polynomial parameters depend on the radius and the turn angle of the circular arc they have to approximate. So, as described by Altafini (1999), "the instantaneous radius of the polar curve is uniquely defined for each turn". Thus, using the information contained in the database (type of road, radius of curvature...), N.A.I.C.C. is able to produce a reference trajectory matching with a given driver profile. Here, the basic idea is to define the polynomial parameters not as functions of the circular arc to be approximated but as functions of the radius of curvature and of the turn angle of the bend which is to be negotiated. Hence, the reference trajectory will be unique considering the bend characteristics.

Contrary to length-dependent curves like clothoids, the polar polynomial curve has a closed-form easy to compute expression. Moreover, it is not subject to mismatching at its end-points because no integration is required for the determination of the Cartesian position $(x, y)$ of the points forming the curve. However, the development of the polynomial presented by Nelson is based on the assumption of a constant speed along the path. This condition is not acceptable in the present study where the speeds before and after the turn are different (different radius of curvature at the junctions with the line-arcs). A later paper described the generalisation of this principle to a robot assuming variable speed (Pinchard, Liegois, \& Pougnet, 1996).

\subsection{Notations}

Table 1 sums up the notations used in this study.

The characteristics of the polar curve are illustrated in Fig. 4. The reference trajectory will be computed in a polar coordinate frame whose centre corresponds to the centre of the turn.

\subsection{Definition of the polar polynomials}

The general expression of the polynomial is given by Eq. (1) where the number of parameters $a_{i}$ defining the polar radius $r$ as a function of the polar angle $\phi$ depends 
Table 1

List of symbols

\begin{tabular}{ll}
\hline$r(t)$ & Radius of curvature of the path \\
$a_{i}$ & Parameters of the polar polynomial \\
$\phi(t)$ & Polar angle \\
$S$ & Path length \\
$\alpha$ & Turn angle of the turn \\
$R$ & Radius of the turn \\
$t$ & Time \\
$v(t)$ or $v$ & Instantaneous speed \\
$R_{i}$ & Radius of the trajectory at a given point $i$ \\
$k$ or $k(t)$ & Curvature of the path \\
\hline
\end{tabular}

on the number of conditions (continuity constraints) imposed:

$r(\phi)=a_{0}+a_{1} \phi+a_{2} \phi^{2}+a_{3} \phi^{3}+\cdots+a_{n} \phi^{n}$.

Satisfying the position, heading and curvature constraints at each end-points as defined by Nelson and with identical initial and final values of the radius (equal to the radius $R$ of the approximated arc of circle) leads to the solution:

$r(\phi)=R\left(1+\frac{\phi^{2}}{2}-\frac{\phi^{3}}{\alpha}+\frac{\phi^{4}}{2 \alpha^{2}}\right)$

obtained with the conditions:

at $\phi=0 \Rightarrow r=R, \quad r^{\prime}=0, \quad k=0$,

at $\phi=\alpha \Rightarrow r=R, \quad r^{\prime}=0, \quad k=0$,

where $r^{\prime}=\mathrm{d} r / \mathrm{d} \phi$ and $k$ represents curvature in the polar frame.
Eq. (2) shows that, for a given couple $(R, \alpha)$ defining the approximated circular arc, a unique solution of the robot path is defined.

In the case of a line-arc-line transition with different speeds and thus different radii ( $R 1$ initial radius and $R 2$ final radius), the parameters $a_{i}$ of the polynomial curve are functions of $R 1, R 2$ and $\alpha$ (Pinchard et al., 1996). As the initial and final postures of the robot used are unknown, Pinchard et al. proposed to take account of dynamic and kinematic constraints in order to define acceptable values of the radii.

In a polar coordinate frame, curvature is defined by the relation (Smirnov, 1972):

$k(\phi)=\frac{\mathrm{d} \theta}{\mathrm{d} s}=\frac{r^{2}+2 r^{\prime} 2-r r^{\prime \prime}}{\left(r^{2}+r^{\prime} 2\right)^{3 / 2}}$,

where $r^{\prime \prime}=\mathrm{d}^{2} r / \mathrm{d} \phi^{2}, \mathrm{~d} s$ is the variation of the path length and $\mathrm{d} \theta$ is the variation of the vehicle's heading whose centre of rotation is following the path. During bend negotiation, this vehicle is subjected to the centripetal force:

$F_{c}=m \frac{v^{2}}{r}=m v^{2} k=m \gamma_{T}$.

Eq. (5) shows that the continuity constraint on curvature induces the continuity of the transverse acceleration $\gamma_{T}$. This reflects directly the driver's behaviour whose aim is to minimise the lateral acceleration variations during curve negotiation. Imposing a peak value of the acceptable centrifugal acceleration for a given kind of driver can then help to determine the reference path (by

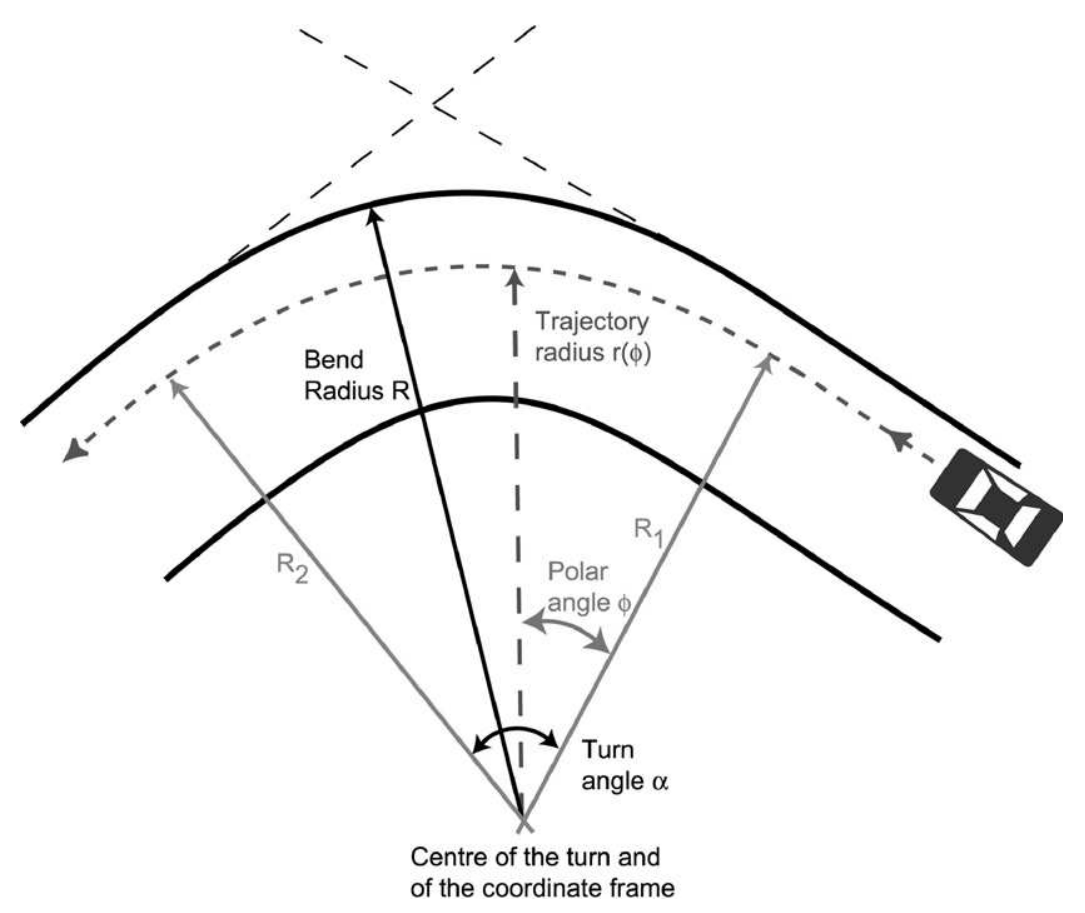

Fig. 4. Characteristics of a polar curve. 
imposing a maximum value of curvature and thus a minimum value of the radius). Moreover, maximum curvature $k_{\max }$ along the path also gives information on the maximum steering angle required to follow this path. Thus, for suitable trajectory modelling, the curvature constraint is primordial.

The speed can be obtained by derivation of the variation of the path length $\mathrm{d} s$ with respect to the time $t$

$v(t)=\frac{\mathrm{d} s}{\mathrm{~d} t}=\left(r(\phi)^{2}+r(\phi)^{\prime 2}\right)^{1 / 2} \frac{\mathrm{d} \phi}{\mathrm{d} t}$.

The analytical expression of the speed allows its online computation. The longitudinal acceleration is obtained by derivation of this expression with respect to the time. Thus, the path-planning algorithm provides a reference trajectory as well as the speed, the heading of the vehicle, curvature, the centrifugal as well as the longitudinal acceleration required to follow this path. Nevertheless, this computation requires a time-varying definition of the polar angle $\phi$ (Pinchard et al., 1996).

\section{Application to trajectory modelling}

In the literature, the curves produced with polar polynomials are intended to be close to circular arcs and symmetric with respect to the line bisecting the turn angle $\alpha$ (Altafini, 1999; Nelson, 1989a). Moreover, the principle consists in generating a smooth transition between two lines, knowing the initial and final postures for the guidepoint of the vehicle. Another application is to determine these postures considering dynamic and kinematic constraints of the robot used (Pinchard et al., 1996). Here, the aim is to use the flexibility of the polar curves and the characteristics depicted above (easy computation of the speed, accelerations...) to precisely and easily model measured trajectories of different kinds of drivers. The shape of the curves can be more or less regular/smooth depending on the number of continuity conditions imposed. So, these curves are well suited for trajectory modelling and for on-line computation when implemented in the driver-aid system.

The results shown here are based on tests carried out with different kinds of drivers in the same conditions in terms of the vehicle used, the turns that have been studied.... The drivers were chosen according to the well-defined classification proposed by Rothengatter et al. (1993). The analysis will consider two driver profiles representative of opposite driving styles: the "novice driver" (ND) and the "very experienced driver" (VED).

\subsection{The "novice driver"}

During bend negotiation, the ND progressively turns the steering wheel, increasing the steering angle to a maximum, then smoothly turns it to the initial point, decreasing the steering angle. This driving technique leads to a progressive and smooth trajectory (cf. Fig. 5) based on a nearly constant lateral acceleration.

Using the polar coordinate frame as defined in Fig. 4, with its centre located on the centre of the turn, and representing the instantaneous radius of curvature of the ND's trajectory with respect to the polar angle (going from 0 to the turn angle $\alpha$ ) leads to Fig. 6 .

The parabolic shape and its symmetry with respect to the vertex (at $\phi=\phi_{C}$ ) is representative of the trajectory progressiveness of a "novice driver". It is also important to note that the heading of the vehicle and thus the slope $r^{\prime}$ at the beginning (at $\left.\phi=0\right)$ and also at the end $(\phi=\alpha)$ of the turns is not equal to zero. This indicates that the driver anticipates the turn, increasing his comfort and safety (Donges, 1978) by smoothing the trajectory. Because of this driving technique, bend negotiation is not totally achieved when the vehicle reaches the end of the turn, as can be seen with the heading value. Consequently, to model the ND more precisely, extra conditions must be added to Pinchard's generalised model:

$$
\begin{aligned}
& \text { at } \phi=0 \Rightarrow r=R_{1}, \quad r^{\prime}=r_{1}^{\prime}, \quad k=k_{1}, \\
& \text { at } \phi=\phi_{C} \Rightarrow r=R_{C}, \quad r^{\prime}=r_{C}^{\prime}, \\
& \text { at } \phi=\alpha \Rightarrow r=R_{2}, \quad r^{\prime}=r_{2}^{\prime}, \quad k=k_{2} .
\end{aligned}
$$

These eight conditions imposed on the position, the heading and on curvature yield a seventh-order polynomial, the eight coefficients $a_{i}$ are therefore only defined as functions of $\alpha, R_{1}, R_{2}, R_{C}, r_{1}^{\prime}, r_{2}^{\prime}$, and $r_{C}^{\prime}$ using the eight conditions.

\subsection{The "very experienced driver"}

A VED makes greater use of the overall capabilities of the vehicle, neglecting the comfort aspect but improving the efficiency of his driving. He is constantly searching for visual information on what will come in front of the car, or what will come after the bend he has to negotiate. This VED behaviour is illustrated in Figs. 7 and 8 which represent, respectively, the measured trajectory and its radius of curvature during the same bend negotiation as the one presented for the "novice driver" (cf. Section 5.1). The shapes of the trajectory and thus of the instantaneous radius of the two kinds of drivers are completely different as can be seen here.

\subsubsection{The "waiting period"}

During the "waiting period" (from $\phi=0$ to $\phi_{P}$ ), the radius of curvature is maintained constant or slightly increasing, which means that the steering angle is constant and near zero. Moreover, an important braking manoeuvre (not illustrated here) is performed at that time. The VED does not anticipate this specific 


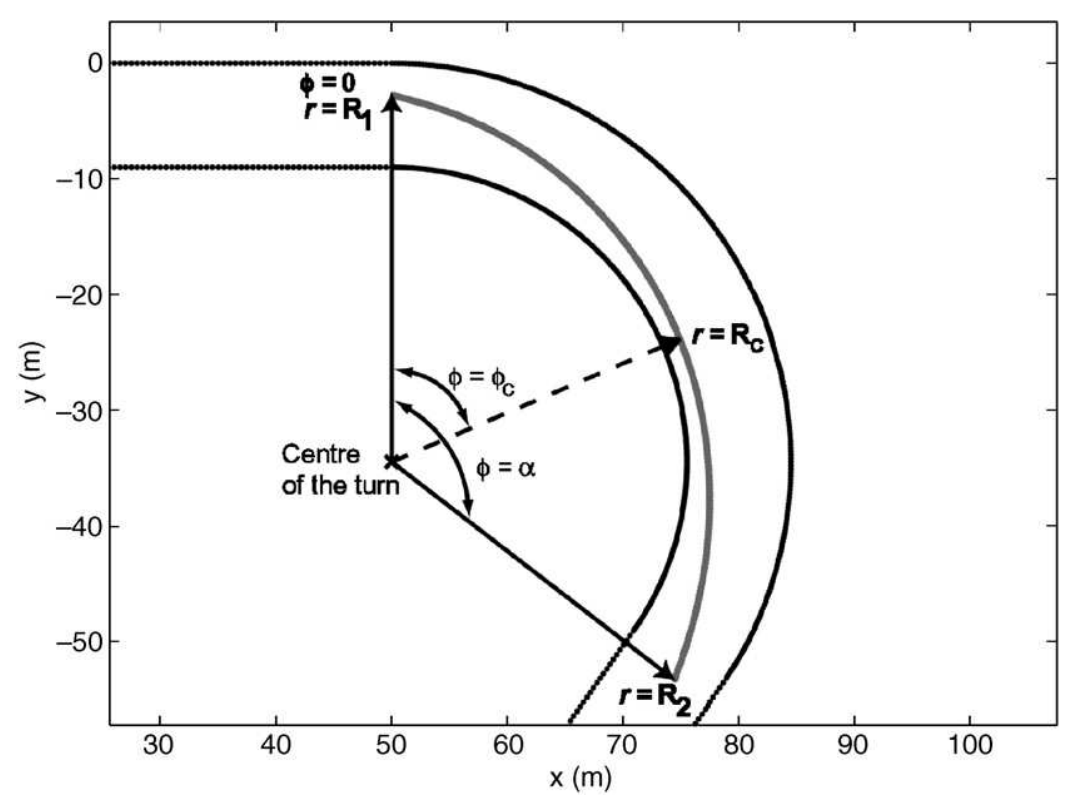

Fig. 5. Measured trajectory of the "novice driver".

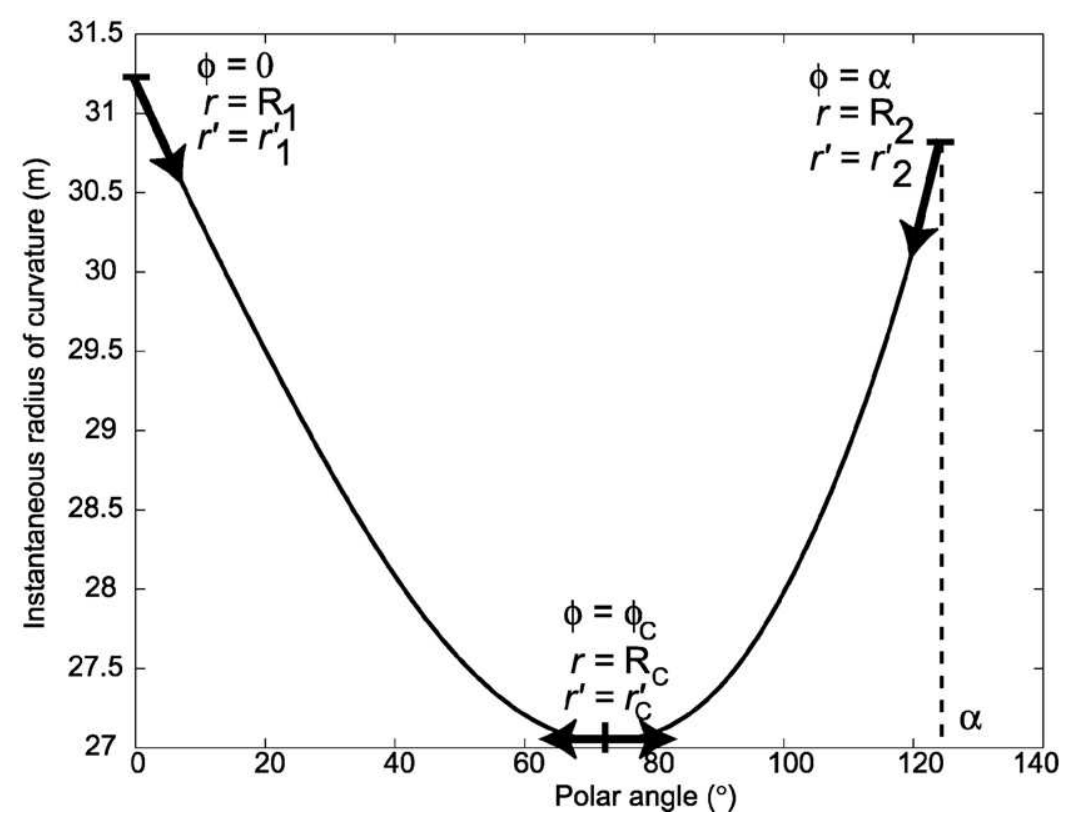

Fig. 6. Instantaneous radius of the "novice driver's" trajectory in the polar frame.

manoeuvre but is searching for some visual information about the end of the bend and about the type of the road profile (straight line, bend...) after this curve. The bend negotiation will then depend on all the information the driver collects during the "waiting period". At the beginning of the turn, the "very experienced driver" is closer to the external side of the road, as can be seen with the value of $R_{1}$ which is greater than the one of the ND (the radius reflects the lateral position of the vehicle in the bend). This allows him a better visual field. Moreover, the value of slope $r_{1}^{\prime}$ (equal to zero) indicates that the bend negotiation did not begin at this given point.

\subsubsection{Reaching the vertex $\left(\phi=\phi_{C}\right)$}

Once the driver is able to see the end of the turn (at $\phi=\phi_{P}$ ) and what is beyond, he rapidly changes the steering angle in order to reach the vertex, which is the nearest point to the centre of the turn (minimum of the radius of curvature). Due to the latter negotiation manoeuvre, the vertex is not located near the middle of the bend but almost at its end. This means that the 


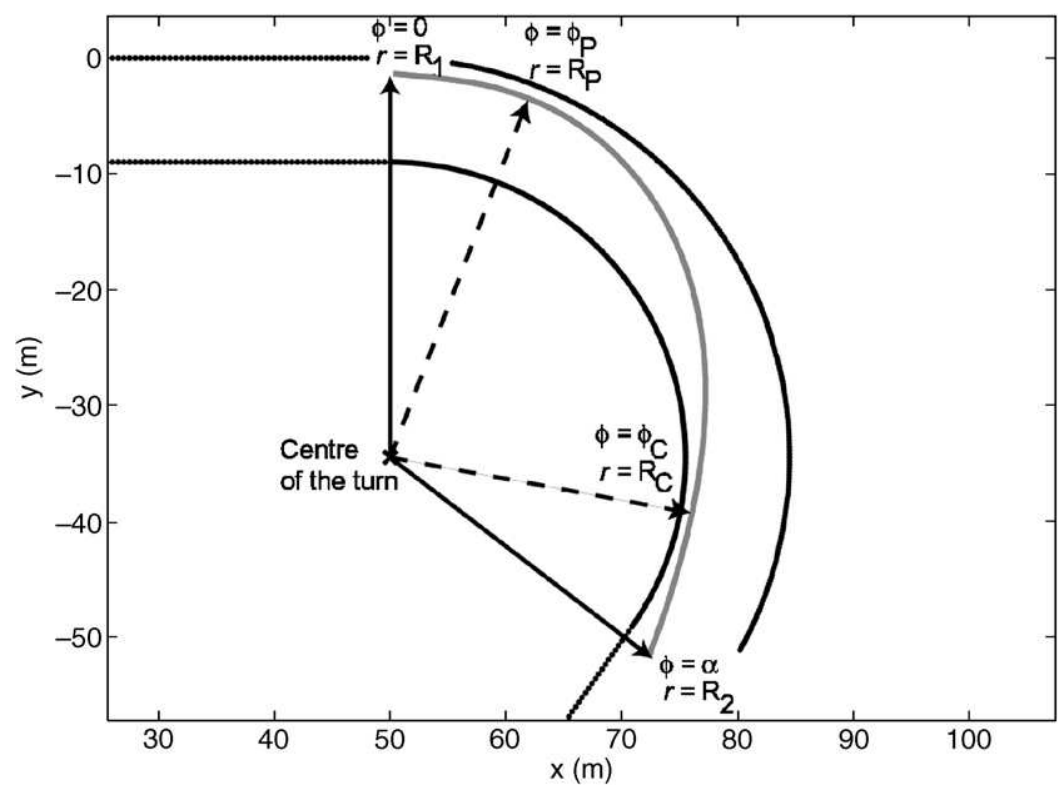

Fig. 7. Measured trajectory of the "experienced driver".

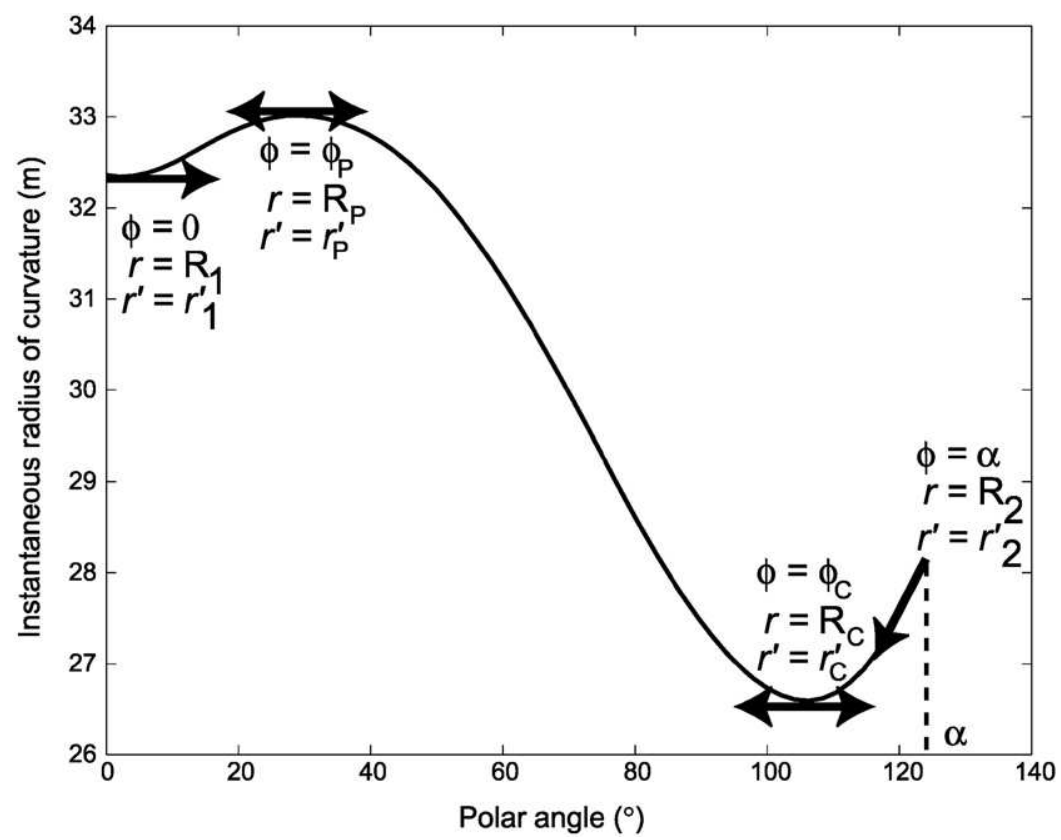

Fig. 8. Instantaneous radius of the VED's trajectory in the polar frame.

vehicle is reaching the end of the turn near the internal side of the road, with a heading angle that allows an early acceleration (before the vertex).

\subsubsection{Driving out of the bend}

The end of the turn is achieved at a higher speed than the one of the "novice driver": this driving technique allows the driver to reaccelerate before reaching the vertex. The value of radius $R_{2}$ shows clearly that the VED is also closer to the internal side of the road and thus, that he benefits from the whole road width to prepare for the next critical driving situation.

This analysis shows that, in order to define a model of the VED path precisely, new conditions are necessary to represent the "waiting period":

at $\phi=0 \Rightarrow r=R^{1}, \quad r^{\prime}=r_{1}^{\prime}, \quad k=k_{1}$,

at $\phi=\phi_{P} \Rightarrow r=R_{P}, \quad r^{\prime}=r_{P}^{\prime}$,

at $\phi=\phi_{C} \Rightarrow r=R_{C}, \quad r^{\prime}=r_{C}^{\prime}$,

at $\phi=\alpha \Rightarrow r=R_{2}, \quad r^{\prime}=r_{2}^{\prime}, \quad k=k_{2}$. 
The path model for a VED, using the conditions defined in Eq. (8), is a ninth-order polar curve.

The values of positions $R_{1}, R_{2},\left(\phi_{C}, R_{C}\right),\left(\phi_{P}, R_{P}\right)$, slopes $r_{1}^{\prime}, r_{2}^{\prime}, r_{C}^{\prime}, r_{P}^{\prime}$ and curvature $k_{1}$ and $k_{2}$ are driverbut also road-dependent (radius of the turn, turn angle, width of the road...). In fact, the driver uses (visual, proprioceptive) information to determine target points for his trajectory. The objective is now to define, for each driver profile, these indicators considering the characteristics of the turn and principally the turn angle and the radius of curvature. As this information is stored in the digital database, the driver-aid system will be able, knowing the driver's profile, to first compute the special points defined above and the parameters of the polar curve, then the whole trajectory.

\section{Parameter identification}

The problem is to know what kind of information the driver's judgement refers to when he determines his trajectory or the longitudinal actions (velocity, acceleration, braking) needed for bend negotiation. It is obvious that the actions applied are dependent on the danger quotation of the situation, but how can this be evaluated? Its evaluation is mainly based on visual information that the driver perceives: is the radius of the curve great or not, is it an open bend or not (little or important turn angle), ....? The driver relies on a subjective estimation of the bend characteristics in order to choose his trajectory (postures) and the associated actions. Thus, the characteristic points $\left(\left(\phi_{C}, R_{C}\right)\right.$, $\left.\left(\phi_{P}, R_{P}\right) \ldots\right)$ presented in the last section can be expressed with respect to the road profile allowing the definition of the parameters $a_{0}, a_{1 \ldots} a_{n}$ of the polar curve for a given driver and a given turn (cf. Fig. 9).

The initial research study showed a linear relation between the position of the characteristic points and the turn radius $R$ in given road characteristics ( $30 \mathrm{~m}<R<150 \mathrm{~m}$ ). This would mean that a driver only considers the bend radius for driving. But this is not the case and can easily be verified with a simple example: the trajectories (in terms of the lateral position of the vehicle on the road) that a driver chooses for two bends with identical radius of curvature but with different turn angles, i.e. a hair-pin vs. an open curve, are completely different. In the case of a VED driving in an open curve allowing him to see beyond its end, the trajectory is comparable to the one of a ND in terms of its progressiveness/shape because there is no need to drive the vehicle near the external side of the road in order to increase the field of view. Thus, the "waiting period" is non-existent. The direct consequence is that for a generalisation of the pathplanning algorithm, the turn angle must be taken into account.

Besides this qualitative study, the goal is now to quantify these results. An extended analysis performed in bends covering a large field of configurations (various radii of curvature and turn angles) showed that the existing relations between the characteristic points and the road profile are highly non-linear. The solution proposed consists in the determination of the polar coordinates of the points reflecting the driver's profile, namely vertex $C\left(\phi_{C}, R_{C}\right)$ for the ND, vertex $C$ and maximum $P\left(\phi_{P}, R_{P}\right)$ for the $\mathrm{VED}$, considering the radius and the turn angle. Knowing the position of these points, the polar radii $R_{1}$ and $R_{2}$ can be determined using a FIS.

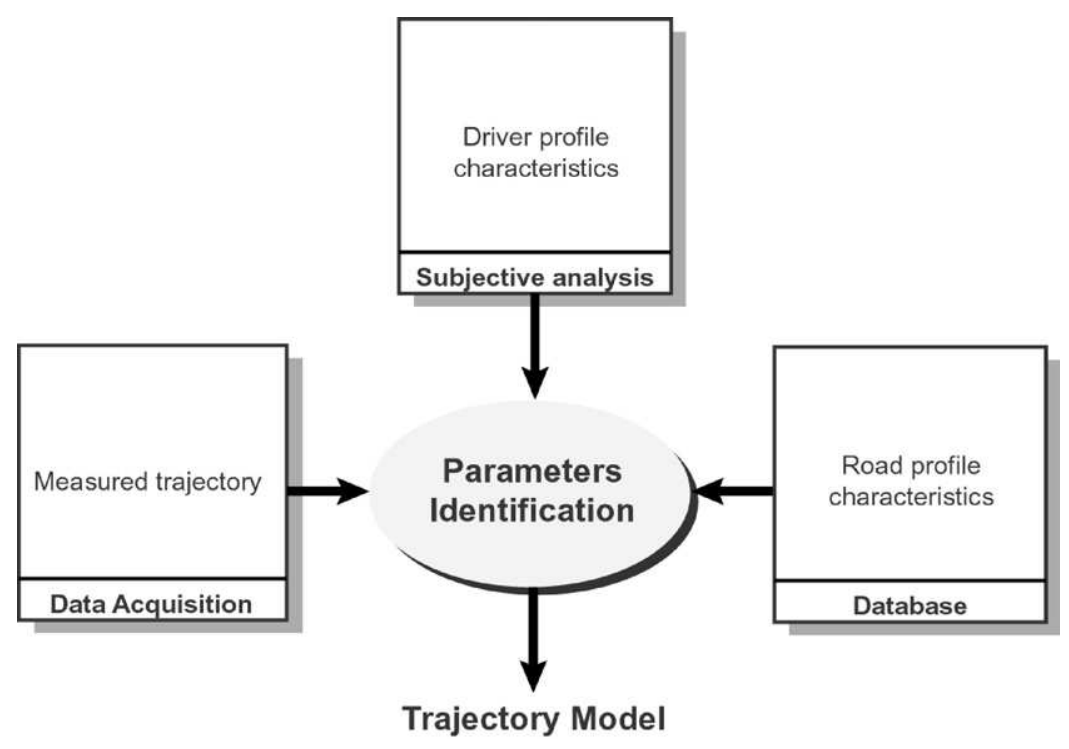

Fig. 9. Identification of the model parameters. 


\subsection{Determination of the driver-dependent points}

The driver-dependent points are in fact reference points chosen by the driver to be targets of his trajectory. Using these targets, the driver determines any actions which he considers necessary to bring his vehicle into good position at these chosen points. A statistical analysis on every bend of the test track and on other road profiles where tests were carried out showed that an ND as well as a VED locate their reference points (in terms of the angular position in the turn) considering the turn angle only. In other words, the polar angles $\phi_{P}$ and $\phi_{C}$ only depend on the turn angle of the bend.

The determination of the instantaneous radius of the trajectory, which reflects the lateral position of the vehicle directly, is a more complex task. It has been shown in Section 6 that the lateral position of the vehicle depends on the radius of curvature but also on the turn angle. Based on a polynomial expression of radii $R_{C}$ and $R_{P}$ with respect to the radius of the turn $R$ and the turn angle $\alpha$, a representation model has been developed. This model has been obtained using an iterative algorithm based on the least square method with quadratic error optimisation. Eq. (9) represents the polynomial and illustrates the non-linearity depicted above mainly by its fourth order, but also by the use of crossed terms (function of $R$ and $\alpha$ ):

$R_{C, P}=A_{0}+\sum_{i=1}^{4} A_{i} R^{i}+\sum_{i=1}^{i=4} B_{i} \alpha^{i}+\sum_{\substack{i=1 \\ j=1}}^{\substack{j=2 \\ i=2}} A_{i j} R^{i} \alpha^{j}$.

\subsection{Determination of the end-points postures}

It appears clearly that the driving task depends on a subjective estimation of the visual, but also proprioceptive perception of external information. During the perception phase, the driver evaluates the danger quotation of the next situation (straight-line driving, curve negotiation, lane-change manoeuvres...) and uses this evaluation to determine his target points. But how does he determine this danger quotation leading to the choice of the appropriate actions? His judgement is largely based on his knowledge and driving experience. An expert-type controller will then be an effective solution to perform an objective quantification of the subjective/qualitative assessment made by the drivers. The fuzzy-logic tool (Zadeh, 1965) is particularly suitable for modelling subjective analyses of non-linear systems and for the representation of the knowledge and experience of a person, a test driver for instance. The particularity of this technique is its capability to treat non-exact ("Large", "High", "Low"...) or badly quantified variables.
As the non-linearity and the subjective character of the vehicle's posture estimation at the end-points have been expressed, a fuzzy-logic system is suited for the determination of these coefficients. The basic idea is to determine $R_{1}$ and $R_{2}$ as functions of the apex $C$ :

$R_{1}=R_{C}+A$,
$R_{2}=R_{C}+B$,

where $A$ and $B$ are the fuzzy variables determined via the fuzzy controller. These fuzzy variables are determined using the radius of curvature and the turn angle of the bend, considering that the driver's behaviour is based on the subjective estimation of these characteristics.

\subsubsection{Structure of the fuzzy inference system (FIS)}

The radius of curvature and the turn angle are the entries of the system which determines the variables $A$ and $B$, using fuzzy rules. A Mamdani or "linguistic" fuzzy controller based on fuzzy propositions both for the antecedents ( $R$ and $\alpha$ ) and the consequents ( $A$ and $B$ ) is used. The antecedents and the consequents are represented with trapezoid and triangular membership functions (cf. Fig. 10) defining the partitioning with linguistic variables of the numerical definition domain. Fuzzy rules using these linguistic variables are developed for the determination of a fuzzy result (i.e. the determination of the consequents' matching linguistic variable). This rule-based computation generates a fuzzy surface representing the possible values of the consequents. The defuzzification, i.e. the computation of the qualitative value of the output, is performed using the centroid method (Bühler, 1994) which consists in the determination of the centre of gravity of the surface generated.

Simulations were carried out to find the best compromise between a satisfactory accuracy of the variable estimation and the complexity of the FIS that is to be implemented in real-time.

\subsubsection{Fuzzy representation of radius $R$ and turn angle $\alpha$}

The implemented membership functions (representation of the radius and the turn angle) allow a fuzzy description of the radius of curvature from 10 to $200 \mathrm{~m}$ and a description of the turn angle from $0^{\circ}$ to $200^{\circ}$. Fig. 10 gives the fuzzy translation of the radius of curvature defined by six membership functions represented by linguistic variables ranging from VERY LOW to VERY IMPORTANT. A degree of appurtenance $\mu$ (also called degree of membership) to a membership function is associated to a given numerical value of the antecedents.

\subsubsection{Fuzzy rules}

Twenty-four rules based on the linguistic variables of the radius and of the angle have been developed to 


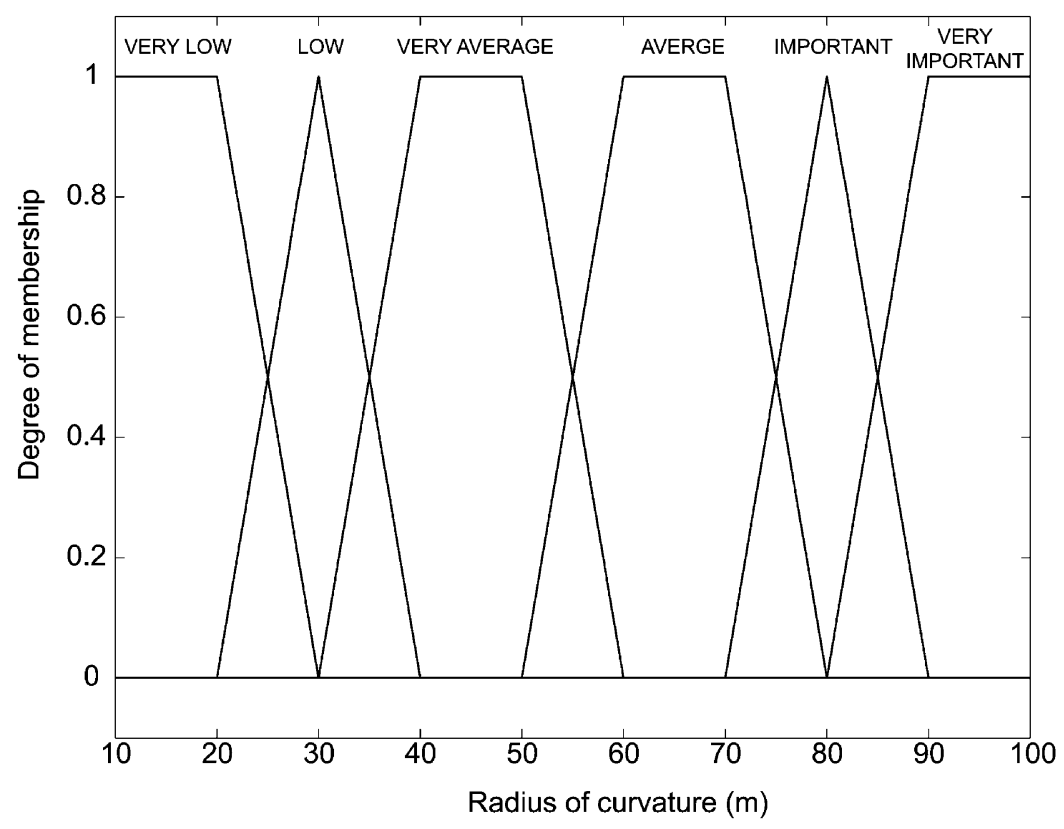

Fig. 10. Fuzzy variables membership of the radius of curvature.

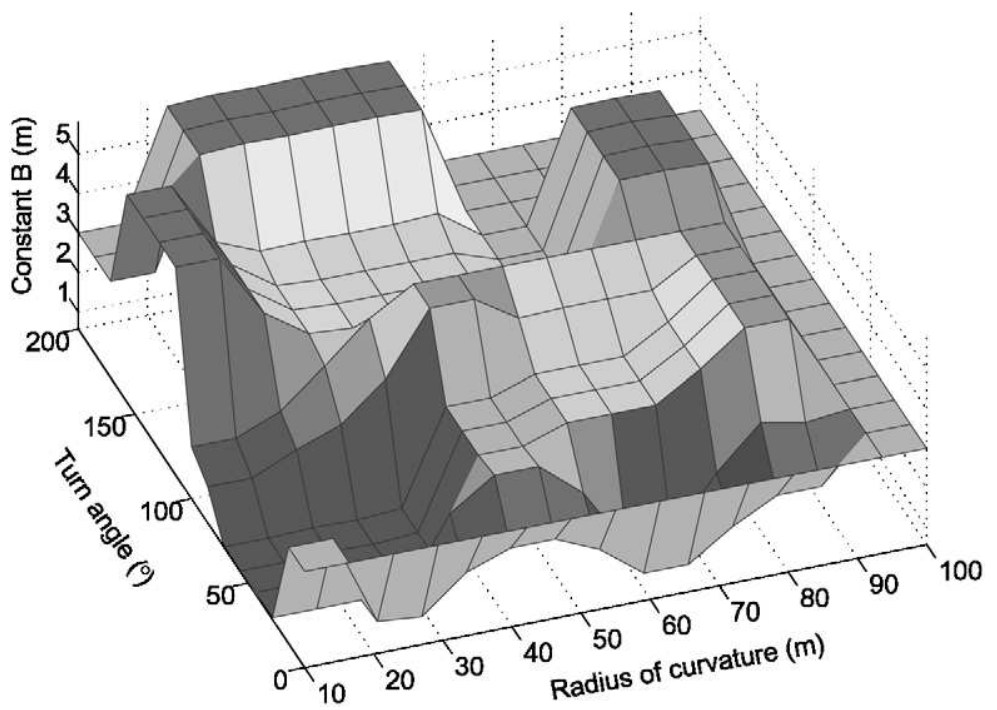

Fig. 11. Three-dimensional representation of the output variables.

determine the value of consequents $A$ and $B$ for a given turn. The general structure of these rules is:

If Radius is VERY LOW and Angle is VERY LOW then Variable is LOW.

\subsubsection{Three-dimensional representation of the output variables}

Given as an example, the resulting estimation of the fuzzy variable $B$ with respect to the radius of curvature and the turn angle is represented in Fig. 11. This illustration shows the evolution of one of the fuzzy controller outputs, as defined by the fuzzy rules, for the antecedents varying in the whole definition domain (from VERY LOW to VERY IMPORTANT). For a given radius of curvature and turn angle, the controller computes the fuzzy variable and, after defuzzification, the radius of the end-point using Eq. (10).

The high non-linearity of this parameter with respect to the characteristics of the turn is obvious, and so is the lateral position (represented by $R_{1}$ and $R_{2}$ ) of the vehicle at the end-points. Taking the example of a bend with a turn angle of $50^{\circ}$, it can easily be noted that the fuzzy variable increases non-linearly (from $0.5 \mathrm{~m}$ to approximately $5 \mathrm{~m}$ ) with the radius of curvature. This means 
that, for this given angle value, the vehicle is nearer and nearer the external side of the road (cf. Eq. (10)) with the increasing radius but the evolution is not constant.

\section{Results}

As the aim of the work presented here is to obtain an accurate model of a driver's behaviour through a mathematical representation of his trajectory during the negotiation of a bend, the first step consists in validating the model presented. This validation, performed on various road profiles (turns with different characteristics) for the ND and the VED, consists in the comparison of the generated polar path with the measured trajectory.

Once the model has been validated, it is possible to develop a complete path planner which processes more complex road profiles than just one turn. This path generator determines, in real-time, the trajectory of the vehicle in a succession of straight lines and turns. Thus, the second part of this section deals with the results of the overall N.A.I.C.C. path-planning module.

\subsection{The polar curve model}

The results presented in this section were carried out with the laboratory test car on the test track. No specific driving conditions were imposed except that the test drivers could use the whole track width. The informations required for the on-line computation of the polynomial parameters were provided by the new database implemented in the N.A.I.C.C. project. During these trials, the lateral controller was used in the "Driver Alarm Mode", that is, the purpose was to validate the real-time capabilities and the precision (in terms of the generated trajectory vs. the real one). The vehicle's trajectory was measured with the DGPS system with a precision of up to $1 \mathrm{~cm}$. The instantaneous radius of curvature of the path was determined with respect to the centre of the turn. Fig. 12 presents the planned path corresponding to the seventh-order polar polynomial curve obtained after computation of the eight parameters (dotted line) and the real path (solid line) in the case of the "novice driver". It can be seen that the estimation of the initial and final postures (positions and headings) of the guide-points and the apex $C$ gives good results.

The tests performed with the "very experienced driver" showed similar results and are not discussed here.

\subsection{The path-planning module}

Finally, a N.A.I.C.C. path-planning algorithm based on the previously presented curve generation method has been developed to compute a reference trajectory in more complex driving situations. Once the vehicle has precisely been located on the database, the actual path-planning module is able to determine a reference path that corresponds to a given driver profile for a succession of straight lines and curves.

The principle of this high-level planner is first to precisely represent the trajectory of the vehicle in the different turns and then to connect these computed

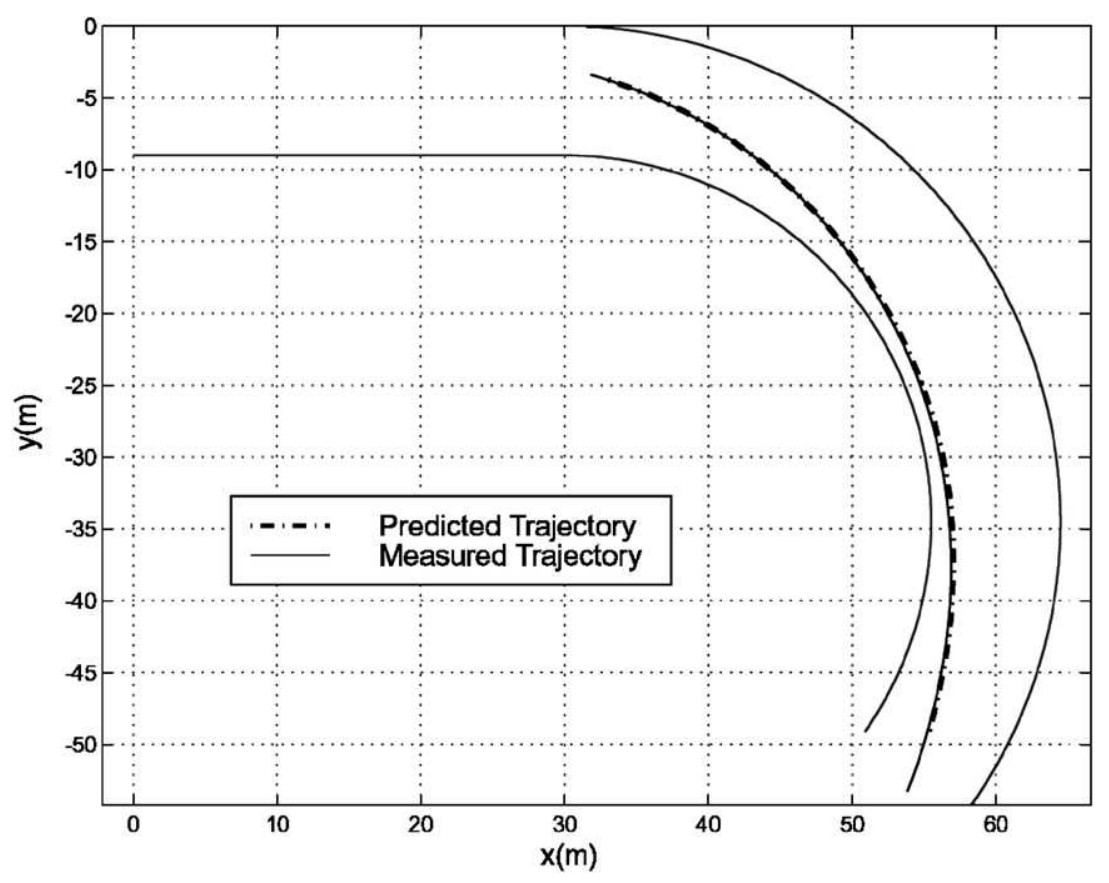

Fig. 12. Measured and planned paths of the "novice driver". 
polar curves using Cartesian polynomials to represent the trajectory in the straight lines. The Cartesian polynomials are based on the continuity conditions imposed for the polar polynomials at the junctions between the straight lines and the turns. The principle is derived from the continuous curvature path generation for lane change manoeuvres (Nelson, 1989a). Figs. 13 and 14 present the results of the N.A.I.C.C. path planner for the driver profiles studied in this paper, in the same 3-bends succession.

The generated ND path very well illustrates the characteristics of this kind of smooth driving based on the search for maximum safety and comfort.

The identified particularities of the VED (see Section 5.2) are also very well represented on the generated trajectory presented in Fig. 14. Before each turn, the

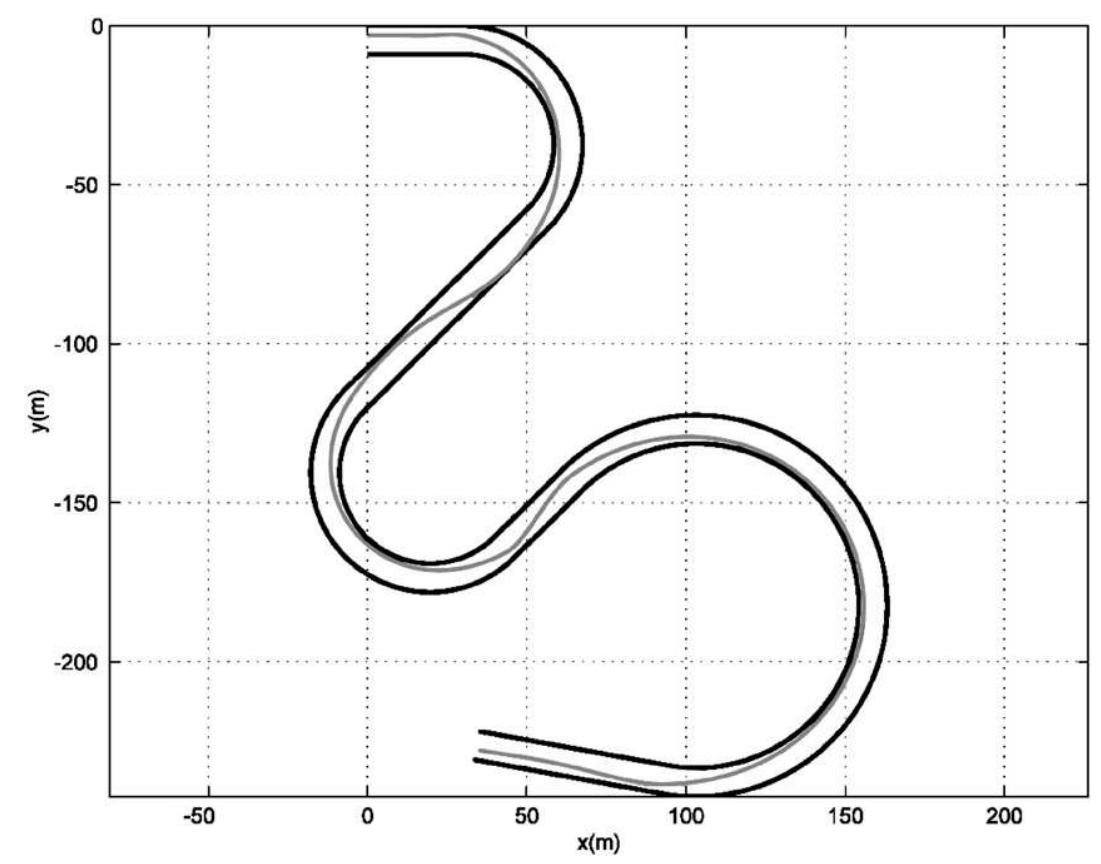

Fig. 13. Planned path for the "novice driver".

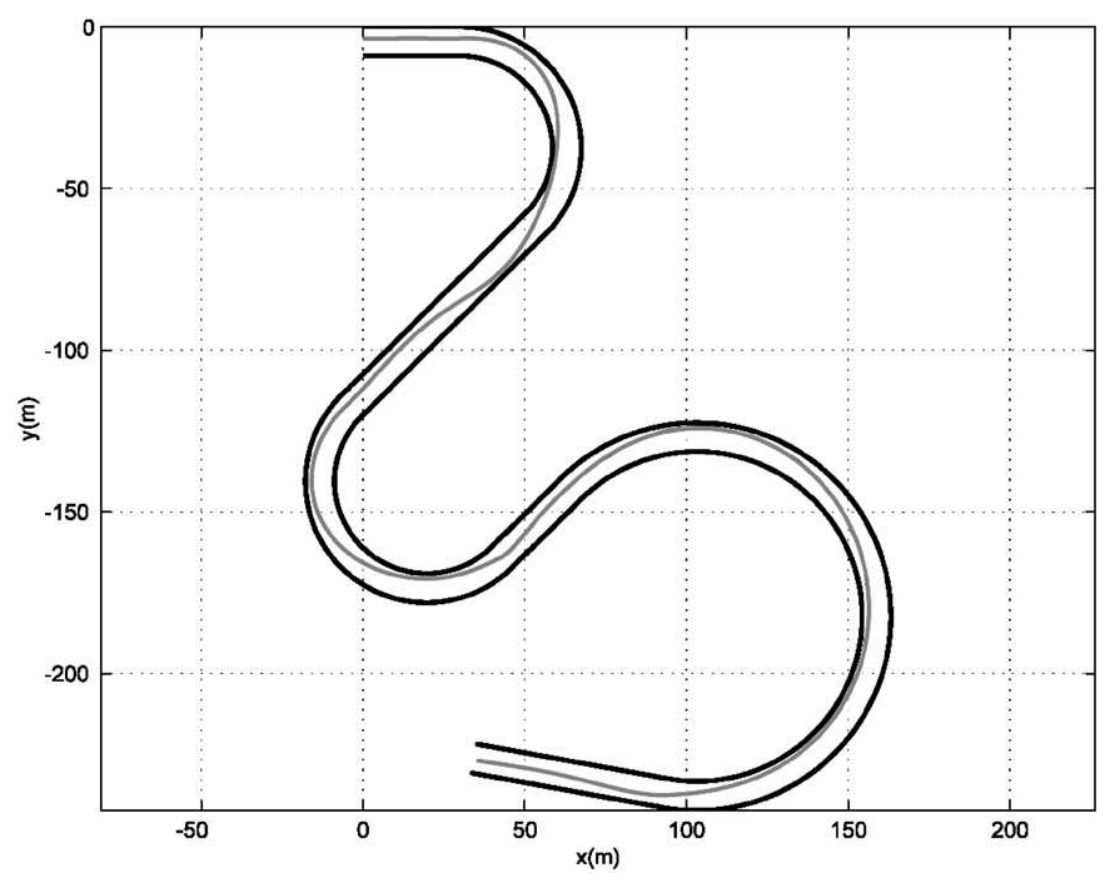

Fig. 14. Planned path for the "very experimented driver". 
vehicle approaches the external roadside (with respect to the centre of the curve). This allows the VED to improve his sight angle but also to brake later than the ND. The position of vertex $C$, near the end of the bend also corresponds to the assumption made during the model structure identification. Finally, a VED makes better use of the overall capabilities of his vehicle (braking capabilities, longitudinal but also lateral potentials, ...).

\section{Conclusion}

This paper has described the concept of a new intelligent driver-aid system, the Navigation Aided Intelligent Cruise Control (N.A.I.C.C.) system. The principle of the lateral controller of the N.A.I.C.C. system is, in the first step, to warn the driver after an early detection of a critical situation in terms of trajectory, and, in the second step, to correct the vehicle's path referring to a calculated model. Once a precise vehicle location has been obtained by data fusion of sensor information, this system determines the distance to the next bend and computes a reference path appropriate to the negotiation of this curve.

The proposed path-planning algorithm is based on polar curves applied to the line-arc-line transition with different speed conditions. On the one hand, these curves provide a continuous curvature along the path, and, on the other hand, across the line-arc junctions. Therefore, the speed and continuous heading angle of the vehicle can be expressed as time-dependent functions. Furthermore, their closed form expression enables real-time computation. The continuous curvature constraint can be observed here as a continuity on the lateral acceleration. This driver system is then capable to generate a reference trajectory which corresponds to the driver behaviour whose aim is to avoid lateral acceleration variations during bend negotiation. The polynomial parameters are fuzzy determined with respect to the database information (radius and turn angle of the bend) and the constraints given by the driver, and thus, are proper to each configuration. The efficiency of the fuzzy determination of the end-points has been highlighted.

This study considers the case of single bends and successions of straight lines and turns. The next step is the extension of this method to the case of successive bends (right-right, left-right bends...), without straight lines connecting them. A succession of bends cannot be considered as two independent bends: an evaluation carried out by test drivers has clearly shown that the trajectory a driver takes in a bend depends on the next driving situation he will meet. In the case of a bend followed by a straight line, the path obtained is the one presented in this paper. In the case of a succession of turns, the driver adapts the negotiation of the first curve in order to achieve the second in suitable conditions, according to his profile.

In the "Driver Alarm Mode" presented, the N.A.I.C.C. can be considered as a warning system, generating a reference path and warning the driver when he deviates too much. But the chosen mathematical model can be used to determine the associated steering angle function and thus to provide a steering controller. In that field of application, it has been shown that linear control structures are possible, but in order to take account of the saturation of the tyres when the vehicle is at the limits of its performance, non-linear controllers are more suitable (Sharp, Casanova, \& Symonds, 2000).

\section{Acknowledgements}

The authors wish to thank Zahir Messaoudene for his interest and contribution to this research work.

\section{References}

Altafini, C. (1999). Curve negotiating using polar polynomials for nonholonomic vehicles. Proceedings of the 14th IFAC World Congress, Vol. Q, Beijing, China, July 5-9.

An, P. E., \& Harris, C. E. (1996). An intelligent driver warning system for vehicle collision avoidance. IEEE Transactions on System, Man and Cybernetics, 26(2), 254-261, ISBN 0018-9472.

Bühler, H. (1994). Réglage par logique floue. Presses polytechniques et universitaires romandes, ISBN 2-88074-271-4.

Donges, E. (1978). A two-level model of driver steering behaviour. Human Factors, 20(6), 691-707.

Gounon, R., \& Barboux, J.-P. (1997). DASSAULT-SERCEL NP's KART technique extended to dual-frequency reception: New applications are made possible. DSNP.

Lauffenburger, J.-Ph., Baujon, J., Basset, M., \& Gissinger, G. L. (2000), The N.A.I.C.C. project: A navigation aided intelligent cruise control system. SAE 2000 Transactions, Journal of Passenger Car-Electronic and Electrical Systems, ISBN 0-7680-0891-3.

Millot, P. (1998). Concepts and limits for human-machine cooperation. Proceedings of the IEEE SMC CESA'98 Conference, Hammamet,Tunisia.

Nelson, W. (1989a). Continuous-curvature paths for autonomous vehicles. Proceedings of the IEEE international conference on robotics and automation, Vol. 3, Scotsdale, pp. 1260-1264.

Nelson, W. (1989b). Continuous steering-function control of robot carts. IEEE International Conference on Industrial Electronics, 36(3), 330-337.

Onken, R. (1993). The compelling evolution of operator assisting systems. In Gärtner Stein Widdel Mensch-Maschine-Systeme und Neue Informationstechnologien. Wachtberg-Werthoven, Germany: FGAN-FAT.

Pinchard, O., Liegois, A., \& Pougnet, F. (1996). Generalized polar polynomials for vehicle path generation with dynamic constraints. Proceedings of the IEEE ICRA'96, Minneapolis, USA, pp. 915920.

Roser, M. M., \& Moissidis, Y. (1998). Advanced driver assistance, warning and control systems. Proceedings of FISITA'98, F98S203, Paris, France. 
Rothengatter, J. A., Alm, H., Kuiken, M. J., Michon, J. A., \& Verwey, W. B. (1993). The driver. In J. A. Michon (Ed.), Generic intelligent driver support. London: Taylor \& Frances.

Sharp, R. S., Casanova, D., \& Symonds, P. (2000). A mathematical model for driver steering control, with design, tuning and performance results. Vehicle System Dynamics, 33, 289-326.

Shladover, S. E. (1998). Intelligent transportation systems (ITS) and the automobile: Recent history and future prospects. Proceedings of FISITA'98, F98S118, Paris, France.
Smirnov, V. (1972). Cours de Mathématiques Supérieures (2 ${ }^{\text {ème }}$ éd.). Mascou: Mir, ISBN 5-03-0000001-1.

Yuhara, N., Tajima, J., Horiuchi, S., Iijima, T., Asanuma, N., \& Ikegaya, M. (1998). Advanced driving support system: Toward human-centered automation. Proceedings of FISITA'98, F98T652, Paris, France.

Zadeh, L. A. (1965). Fuzzy sets. Information and Control, 8(3), 338-353.

Zhao, Y. (1997). Vehicle location and navigation systems. Boston, London: Artech House Publisher, ISBN 0-89006-861-5. 